\title{
SOLVABLE GROUPS ACTING ON THE LINE
}

BY

\author{
J. F. PLANTE ${ }^{1}$
}

\begin{abstract}
Actions of discrete groups on the real line are considered. When the group of homeomorphisms is solvable several sufficient conditions are given which guarantee that the group is semiconjugate to a subgroup of the affine group of the line. In the process of obtaining these results sufficient conditions are also determined for the existence of invariant (quasi-invariant) measures for abelian (solvable) groups acting on the line. It turns out, for example, that any solvable group of real analytic diffeomorphisms or a polycyclic group of homeomorphisms has a quasi-invariant measure, and that any abelian group of $C^{2}$ diffeomorphisms has an invariant measure. An example is given to show that some restrictions are necessary in order to obtain such conclusions. Some applications to the study of codimension one foliations are indicated.
\end{abstract}

Introduction. If $G$ is a group of homeomorphisms of the line it is reasonable to ask for a dynamic description of how $G$ acts when reasonable restrictions are placed on $G$. For example, in [9] it is shown that if $G$ is finitely generated and nilpotent then there is a $G$-invariant Borel measure on $\mathbf{R}$ which is finite on compact sets. This already says much about $G$. The proof is quite different from classical arguments which guarantee the existence of a finite invariant measure when an amenable group acts on a compact Hausdorff space [6] in that one uses pseudogroup properties of $G$ rather than group properties (specifically, nonexponential growth of orbits rather than nilpotence of $G$ ). It is reasonable to determine what can be said when $G$ is solvable, but, as pointed out in [9], solvable groups need not have invariant measures, e.g., subgroups of the affine group which contain nontrivial translations and nontrivial dilations.

Such examples suggest consideration of a more general invariance property. A measure is called quasi-invariant (for $G$ ) if each element of $G$ multiplies the measure by a nonzero constant which depends on the particular element of $G$. Usual Lebesgue measure is quasi-invariant for the affine group so it is reasonable to ask if every (finitely generated) solvable $G$ has a quasi-invariant measure. It turns out that a large class of solvable groups (including polycyclic groups) acting on the line must have quasi-invariant measures but there are solvable groups which do not have such measures. An important step in the investigation of solvable groups is the consideration of infinitely generated abelian groups. If differentiability is assumed, stronger

Received by the editors October 30, 1980 and, in revised form, August 3, 1982.

1980 Mathematics Subject Classification. Primary 57E25; Secondary 58F11, 58F15, 58F18.

Key words and phrases. Solvable groups, invariant measures, translation number, quasi-invariant measures, codimension one foliations.

${ }^{1}$ This research was partially supported by a grant from the National Science Foundation. 
results follow. For example, an arbitrary abelian group of $C^{2}$ diffeomorphisms of the line has an invariant measure and an arbitrary solvable real analytic group acting on the line has a quasi-invariant measure. These results are presented in $\S \S 3$ and 4 after basic results concerning existence and uniqueness of invariant measures are established in $\$ \S 1$ and $2 . \$ 5$ describes an example to show that certain hypotheses made earlier were essential. The last two sections contain applications to the study of codimension one foliations. $\$ 6$ considers foliations covered by a foliation with leaf space homeomorphic to $\mathbf{R}$. These results can be used to give an alternate proof of some results in [10]. $\$ 7$ describes a generalization of the Reeb Stability Theorem to continuous codimension one foliations in a neighborhood of a compact leaf having a reasonably nice solvable fundamental group.

1. Fixed points and invariant measures. In this section we recall some results which give sufficient conditions for the existence of an invariant measure. For the necessary background on these results see [6 and 9].

First we consider a locally compact topological group $G$ acting on a compact Hausdorff space $X$ so that the map $G \times X \rightarrow X$ given by $(g, x) \rightarrow g(x)$ is continuous. $C(X)$ is the space of continuous functions from $X$ to $\mathbf{R}$ and $C^{*}(X)$ is the space of bounded $\mathbf{R}$-linear functionals on $C(X)$. By the Riesz Representation Theorem each element of $C^{*}(X)$ is determined by a unique finite (signed) Borel measure as follows: If $\mu$ is the measure and $f \in C(X)$ then the functional is given by $f \rightarrow \int_{X} f d \mu$. Thus, in order to find a $G$-invariant measure on $X$ we consider the induced action $G \times C^{*}(X) \rightarrow C^{*}(X)$ given by $(g, \phi) \rightarrow \phi \circ g$. This action is linear and a $G$ invariant finite Borel measure on $X$ corresponds to a fixed point for this induced action. If $G$ is amenable then any continuous linear action on a compact convex set in a locally convex linear topological space has a fixed point [6, p. 55]. Since the functionals which determine probability measures constitute a compact set in the weak* topology we have

1.1 TheOREM. If $G$ is an amenable locally compact topological group acting continuously on a compact Hausdorff space then there is a G-invariant Borel measure $\mu$ on $X$ such that $\mu(X)=1$.

When the space $X$ is not compact stronger restrictions must be made on $G$ in order to guarantee the existence of $G$-invariant Borel measure. Suppose $G$ is generated by a finite set $G^{1} \subset G$ which is symmetric (i.e., $g \in G^{1}$ implies $g^{-1} \in G^{1}$ ). Let $G^{n}$ be the set consisting of all elements of $G$ which can be written as words of length $\leqslant n$ in the elements of $G$. We say that $G$ has nonexponential growth if

$$
\liminf _{n \rightarrow \infty} \frac{1}{n} \log \left|G^{n}\right|=0 \quad(|| \text { denotes cardinality })
$$

Let $\mathcal{H}(\mathbf{R})$ be the group of homeomorphisms of $\mathbf{R}$ and $\mathcal{H}^{+}(\mathbf{R})$ the subgroup consisting of orientation preserving homeomorphisms. Let $G \subset \mathcal{H}(\mathbf{R})$ be a subgroup. The orbit of $x \in \mathbf{R}$ is the set $G(x)=\{g(x) \mid g \in G\}$. Suppose $G$ is generated by a 
finite symmetric set $G^{1} \subset G$. Define $G^{n}(x)$ to be the set $\left\{g(x) \mid g \in G^{n}\right\}$. We say that the orbit $G(x)$ has nonexponential growth if $\liminf _{n \rightarrow x} \frac{1}{n} \log \left|G^{n}(x)\right|=0$. If $G$ has nonexponential growth as an abstract group then $G(x)$ has nonexponential growth of every $x \in \mathbf{R}$. We denote by Fix $G$ the set of all $x \in \mathbf{R}$ such that $g(x)=x$ for every $g \in G$. If $x \in$ Fix $G$ then $G(x)$ trivially has nonexponential growth.

Suppose $G$ is a subgroup of $\mathcal{H}(\mathbf{R})$ and $\mu$ is a nontrivial $G$-invariant Borel measure on $\mathbf{R}$ with the property that the measure of any compact set is finite. If $G \subset \mathcal{H}^{+}(\mathbf{R})$ we have the translation number homomorphism [9] $\tau_{\mu}: G \rightarrow \mathbf{R}$ given by

$$
\tau_{\mu}(g)= \begin{cases}\mu([x, g(x))) & \text { if } g(x)>x, \\ 0 & \text { if } g(x)=x, \\ -\mu([g(x), x)) & \text { if } g(x)<x .\end{cases}
$$

This definition of $\tau_{\mu}$ does not depend on $x$ and $\tau_{\mu}$ is easily shown to be a homomorphism. Since bounded sets have finite measure it follows that $\tau_{\mu}(g) \neq 0$ if, and only if, for each $x \in \mathbf{R}$ the set $\left\{g^{n}(x)\right\}$ is unbounded in both directions and that $\tau_{\mu}(g)=0$ if, and only if, $\operatorname{Fix}(g)=\{x \mid g(x)=x\}$ is nonempty. (Note that $\tau_{\mu}(g)=0$ implies that $\operatorname{Fix}(g)$ contains the support of $\mu$.) These observations may be summarized as follows.

1.2 Lemma. If $G \subset \mathcal{H}^{+}(\mathbf{R})$ and $\tau_{\mu}: G \rightarrow \mathbf{R}$ as above then $\tau_{\mu}=0$ if, and only if, Fix $G \neq \varnothing$.

We now face the questions of existence and uniqueness of invariant measures for a group $G \subset \mathcal{H}(\mathbf{R})$. The following basic result is implicit in [9].

1.3 TheOREM. Suppose $G \subset \mathcal{H}^{+}(\mathbf{R})$ is a finitely generated group. Then there exists a nontrivial G-invariant measure $\mu$ (which is finite on compact sets) if, and only if, $G(x)$ has nonexponential growth for some $x \in \mathbf{R}$.

Proof. The "if" part is the proof of (5.4) of [9] when Fix $G=\varnothing$ (if $x \in$ Fix $G$ let $\mu$ be the measure $\mu(E)=1$ if $x \in E$ and $\mu(E)=0$ otherwise). The "only if" part is an obvious analogue of (6.3) of [9].

REMARK. It is worth noting that the averaging done in the proof of (5.4) of [9] is carried out on the level of pseudogroups and is somewhat different from the "group-averaging" used to prove results like 1.1 above [6]. Although an analogue of the Følner condition (for discrete groups) can be used to get invariant measures for pseudogroups [5], this condition is not hereditary, i.e., the condition may not be satisfied by some subpseudogroup. Growth properties (e.g., nonexponential growth) are hereditary and this fact is crucial to the proof of 1.3 .

2. Uniqueness of invariant measures. Our next step is to determine the extent to which invariant measures are unique for subgroups of $\mathcal{H}(\mathbf{R})$. Since any nontrivial measure can be multiplied by any positive number to obtain another invariant measure we do not expect strict uniqueness. It is also clear that uniqueness in the extended sense does not occur. For example, if $G$ is the infinite cyclic group generated by the translation $g(x)=x+1$ then an arbitrary finite measure on an 
interval of length one may be translated via the iterates of $g$ to give a $g$-invariant measure on $\mathbf{R}$.

2.1 Proposition. If $G \subset \mathcal{H}^{+}(\mathbf{R})$ and $\mu, \nu$ are two $G$-invariant Borel measures on $\mathbf{R}$ then $\tau_{\mu}$ and $\tau_{\nu}$ are linearly dependent elements of $\operatorname{Hom}(G, \mathbf{R})$.

Proof. If either measure is trivial, the conclusion follows immediately, so we assume $\mu$ and $\nu$ are both nontrivial. By 1.2 the subgroups $\operatorname{ker} \tau_{\mu}$ and $\operatorname{ker} \tau_{\nu}$ are equal since they are characterized by a property independent of the measures. Let $H$ denote this subgroup and let $\bar{\tau}_{\mu}$ and $\bar{\tau}_{\nu}$ denote the elements of $\operatorname{Hom}(G / H ; \mathbf{R})$ induced by $\tau_{\mu}$ and $\tau_{\nu}$, respectively. We must show that $\bar{\tau}_{\mu}$ and $\bar{\tau}_{\nu}$ are linearly dependent. Let $x \in$ Fix $H$. Then the abelian group $G / H$ acts freely and transitively on $G(x)$. Thus, $G / H$ has an Archimedean ordering defined by $g_{1} H<g_{2} H$ iff $g_{1}(x)<g_{2}(x)$. Clearly the homomorphisms $\bar{\tau}_{\mu}$ and $\bar{\tau}_{\nu}$ are order preserving and so must be linearly dependent. This proves 2.1 .

As noted earlier the invariant measure need not be unique when $\tau_{\mu}(G)=0$ or $\tau_{\mu}(G) \cong \mathbf{Z}$. The following result gives uniqueness in other cases.

2.2 Proposition. Suppose $G \subset \mathfrak{H}^{+}(\mathbf{R})$ and $\mu, \nu$ are G-invariant Borel measures such that $\tau_{\mu}(G)$ and $\tau_{\mu}(G)$ are dense in $\mathbf{R}$. Then there is a constant $c>0$ such that $\nu=c \mu$.

Proof. Because of 2.1 we may multiply $\mu$ by a constant and assume that $\tau_{\mu}=\tau_{\nu}$. We must show that $\mu=\nu$ in this case. First we observe that these measures have no atoms, that is, the measure of a singleton is always zero. If $\mu(\{x\})>0$ for some $x \in \mathbf{R}$ then every nontrivial element of $\tau_{\mu}(G)$ would have absolute value at least $\mu(\{x\})$ which would contradict the hypothesis that $\tau_{\mu}(G)$ is a dense subgroup of $\mathbf{R}$. Similarly for $\nu$. Note also that the support sets for $\mu$ and $\nu$ are perfect (otherwise there would be atoms). Since $\mu$ has no atoms the map $h_{\mu}: \mathbf{R} \rightarrow \mathbf{R}$ defined by

$$
h_{\mu}(x)= \begin{cases}\mu([0, x]), & x \geqslant 0, \\ -\mu([x, 0]), & x \leqslant 0,\end{cases}
$$

is continuous and surjective. For each $x \in \mathbf{R}, h_{\mu}^{-1}(x)$ is either a single point in the support of $\mu$ or a closed interval having $\mu$-measure zero and whose endpoints are in the support of $\mu$. Thus, $h_{\mu}$ is a continuous semiconjugacy between $G$ and the group of translations of $\mathbf{R}$ by elements of $\tau_{\mu}(G)$. This means there is a commuting diagram

$$
\begin{array}{ccc}
\qquad \times \mathbf{R} & \stackrel{\alpha}{\rightarrow} & \mathbf{R} \\
\tau_{\mu} \times h_{\mu} \downarrow & & \downarrow h_{\mu} \\
\tau_{\mu}(G) \times \mathbf{R} & \stackrel{\beta}{\rightarrow} & \mathbf{R}
\end{array}
$$

where $\alpha(g, x)=g(x)$ and $\beta(t, x)=t+x$. Since the action $\beta$ is minimal (i.e., every orbit is dense) it follows that the restriction of $\alpha$ to the support of $\mu$ is minimal. Similar considerations are valid for $\nu$. 
In order to show that $\mu=\nu$ we show first that their supports coincide. Suppose $x \in \operatorname{supp} \mu$ but $x \notin \operatorname{supp} \nu$. Since supp $\mu$ is perfect and $G(x)$ is dense in supp $\mu$ there is a $g \in G$ such that $g(x) \neq x$ and the closed interval with endpoints $x$ and $g(x)$ has $\nu$-measure zero. Then we would have $\tau_{\mu}(g) \neq 0$ and $\tau_{\nu}(g)=0$ contradicting the fact that $\tau_{\mu}=\tau_{\nu}$. So $\operatorname{supp} \mu=\operatorname{supp} \nu$. In order to show that $\mu=\nu$ it remains to be shown that they agree on any interval whose endpoints lie in the common support set. Suppose $x<y$ are in the support set. By the minimality of $G$ on this set there is a sequence of elements $g_{n} \in G$ such that $\lim _{n \rightarrow \infty} g_{n}(x)=y$. We have

$$
\begin{aligned}
\mu([x, y)) & =\lim _{n \rightarrow \infty} \mu\left(\left[x, g_{n}(x)\right)\right)=\lim _{n \rightarrow \infty} \tau_{\mu}\left(g_{n}\right) \\
& =\lim _{n \rightarrow \infty} \tau_{\nu}\left(g_{n}\right)=\lim _{n \rightarrow \infty} \nu\left(\left[x, g_{n}(x)\right)\right)=\nu([x, y)) .
\end{aligned}
$$

This completes the proof of 2.2 .

3. Abelian groups and invariant measures. Since solvable groups are built up from abelian groups we consider the problem of finding invariant measures for abelian subgroups of $\mathcal{H}(\mathbf{R})$. Since finitely generated abelian groups have nonexponential growth they always have invariant measures according to 1.3. We now consider what happens when the abelian groups are not finitely generated.

3.1 Proposition. Suppose $G \subset \mathcal{H}(\mathbf{R})$ is an abelian group. If there exists $g \in G$ such that $\operatorname{Fix}(g)=\varnothing$ then there exists a nontrivial G-invariant Borel measure on $\mathbf{R}$ which is finite on compact sets.

Proof. The quotient space $S=\mathbf{R} / x \sim g(x)$ is homeomorphic to the circle and since $G$ is abelian there is an induced action of $G_{g}=G /(g)$ on $S$ (where $(g)$ is the infinite cylic group generated by $g$ ). Since abelian groups are amenable there exists a finite $G_{g}$-invariant measure on $S$ by 1.1 . This measure lifts to a $G$-invariant measure on $\mathbf{R}$.

3.2 CoRollary. If $G \subset \mathcal{H}(\mathbf{R})$ is a fixed point free group (i.e., $\operatorname{Fix}(g)=\varnothing$ for every $g \neq$ identity) then there is a nontrivial G-invariant Borel measure on $\mathbf{R}$ which is finite on compact sets.

Proof. There is an Archimedean ordering on $G$ defined by $g_{1}<g_{2}$ if $g_{1}(x)<g_{2}(x)$ for some (and, therefore, for every) $x \in \mathbf{R}$. By Hölder's theorem [2,p. 226] $G$ is abelian so 3.2 follows from 3.1 .

Later on we will give an example of a countable abelian subgroup of $\mathcal{H}^{+}(\mathbf{R})$ which does not have an invariant measure. For the moment we consider properties that such an example must have. Let $G \subset \mathcal{H}^{+}(\mathbf{R})$ be a group and $F \subset \mathbf{R}$ a $G$-invariant set. We will say that $G$ is boundedly generated on $F$ if there is a symmetric generating set $G^{1} \subset G$ such that $G^{1}(x)$ is bounded for every $x \in F$. Note that $G$ is boundedly generated on $\mathbf{R}$ iff $G$ is boundedly generated on a $G$-invariant set $F$ such that $\inf F=-\infty$ and $\sup F=+\infty$. $G$ is boundedly generated if it is boundedly generated on some nonempty $F$. Clearly any finitely generated subgroup of $\mathcal{H}^{+}(\mathbf{R})$ is boundedly generated on $\mathbf{R}$. We say that a group has property $P$ locally or is locally $P$ if every finitely generated subgroup has property $P$. For example, 
nilpotent groups (or even locally nilpotent groups) have nonexponential growth locally [14].

3.3 Lemma. Let $G \subset \mathcal{H}^{+}(\mathbf{R})$ be a group which is boundedly generated on the $G$-invariant set $F$ by the symmetric generating set $G^{1} \subset G$. Suppose that $H(x)$ has nonexponential growth whenever $x \in F$ and $H \subset G$ is a finitely generated subgroup. Then there is a nontrivial G-invariant Borel measure which is finite on compact sets.

3.4 Corollary. If $G \subset \mathcal{H}^{+}(\mathbf{R})$ is a abelian group which is boundedly generated then there is a nontrivial G-invariant Borel measure on $\mathbf{R}$ which is finite on compact sets.

Proof of 3.3. If $x \in$ Fix $G$ then the measure given by $\mu(E)=1$ if $x \in E$ and $\mu(E)=0$ otherwise, is $G$-invariant and finite. So assume now that Fix $G=\varnothing$. This means that for every $x \in \mathbf{R}$, sup $G(x)=\infty$, inf $G(x)=-\infty$. Now if $G^{1}\left(x_{0}\right)$ is bounded this implies that each orbit of $G$ intersects the closed interval [inf $G^{1}\left(x_{0}\right)$, sup $G^{1}\left(x_{0}\right)$ ] so there exists a closed interval $K$ such that every $G$-orbit meets the interior of $K$. Let $\left\{G_{i}\right\}$ be the collection of all finitely generated subgroups of $G$. For each $G_{i}$ there is a $G_{i}$-invariant Borel measure $\mu_{i}$ such that $\mu_{i}(K)=1$ (since the support of any $G_{i}$-invariant measure intersects $\left.K\right)$. Let $\Lambda_{i} \subset C^{*}(K)$ be the compact convex set corresponding to the set of all such measures. The set $\Lambda=\cap \Lambda_{i}$ is nonempty and its elements correspond to $G$-invariant Borel measures $\mu$ such that $\mu(K)=1$. This proves 3.3.

3.5 Proposition. Suppose $G \subset \mathcal{H}^{+}(\mathbf{R})$ is an abelian group and $H \subset G$ is a nontrivial boundedly generated subgroup such that Fix $H=\varnothing$. Then there exists a nontrivial G-invariant Borel measure on $\mathbf{R}$ which is finite on compact sets.

Proof. By 3.4 there exists a nontrivial $H$-invariant Borel measure $\mu$ on R. Since Fix $H=\varnothing, \tau_{\mu}: H \rightarrow \mathbf{R}$ is nonzero by 1.2 . Therefore, there exists $g \in H$ such that $\operatorname{Fix}(g)=\varnothing$, so it follows from 3.1 that $G$ has a nontrivial invariant measure.

Denote by $\operatorname{Diff}^{k}(\mathbf{R})$ the subset of $\mathcal{H}(\mathbf{R})$ consisting of homeomorphisms which are $k$ times continuously differentiable and by $\operatorname{Diff}_{+}^{k}(\mathbf{R})$ the corresponding subgroup of $\mathcal{H}^{+}(\mathbf{R})$.

3.6 THEOREM. If $G \subset \operatorname{Diff}_{+}^{2}(\mathbf{R})$ is abelian then there exists a nontrivial G-invariant Borel measure which is finite on compact sets.

Proof. Suppose $G$ does not have any invariant measures. According to 3.5, Fix $H \neq \varnothing$ for every finitely generated subgroup $H$ of $G$. On the other hand we have

$$
\bigcap \operatorname{Fix} H=\operatorname{Fix} G=\varnothing
$$

where the intersection is taken over all finitely generated subgroups. Let $H_{0}$ be a nontrivial finitely generated subgroup of $G$ and let $x_{0} \in \mathbf{R}$ be a boundary point of Fix $H_{0}$. Let $H_{1}$ be another finitely generated subgroup of $G$ which contains $H_{0}$ and such that $h_{1}\left(x_{0}\right) \neq x_{0}$ for some $h_{1} \in H_{1}$. Let $a=\inf \left\{h_{1}^{n}\left(x_{0}\right) \mid n \in \mathbf{Z}\right\}$ and $b=$ $\sup \left\{h_{1}^{n}\left(x_{0}\right) \mid n \in \mathbf{Z}\right\}$. Note that either $a$ or $b$ is finite and fixed by $h_{1}$. Without loss of 
generality suppose $a$ is finite. Choose $h_{0} \in H_{0}$ such that $h_{0}\left(x_{1}\right) \neq x_{1}$ for some $x_{1} \in(a, b)$. Since $h_{0}$ and $h_{1}$ commute, $a$ (and $b$ if finite) are fixed by $h_{0}$ and $h_{1}$. So it follows from a result of Kopell [7] that $h_{0}(x)=x$ for every $x \in[a, b)$. This contradicts the fact that $h_{0}\left(x_{1}\right) \neq x_{1}$ and completes the proof of 3.6.

REMARKs. (1) The proofs of 3.5 and 3.6 used the translation number so it was assumed that all elements of $G$ were orientation preserving. This hypothesis can be dropped, however, by the following argument. Let $G^{+} \subset G$ be the subgroup consisting of orientation preserving homeomorphisms. $G^{+}$has index at most 2 in $G$. Let $\mu^{+}$ be an invariant measure given by 3.5 or 3.6. Let $g$ be any orientation reversing element of $G$ and let $\mu^{-}$be the measure induced from $\mu^{+}$by $g$. The measure $\mu=\mu^{+}+\mu^{-}$is nontrivial and $G$-invariant.

(2) 3.5 applies to some groups which are not finitely generated. For example, if $G \subset \mathcal{H}^{+}(\mathbf{R})$ is isomorphic to a subgroup of $\mathbf{Q}$ then $G$ is boundedly generated. This follows immediately from the fact that any subgroup of $G$ has a generating set $G^{1}$ and an infinite cyclic subgroup $H$ with the property that if $g \in G^{1}$ then there is a nonnegative integer $n$ such that $g^{n}$ is a generator of $H$.

We say that $g \in \mathcal{H}(\mathbf{R})$ has isolated fixed points if either $g$ is the identity or $\operatorname{Fix}(g)$ is discrete. $G \subset \mathcal{H}(\mathbf{R})$ has isolated fixed points if every element of $G$ does. For example, elements of $\mathcal{H}(\mathbf{R})$ which are real analytic have isolated fixed points. The proof of the following result is similar to that of 3.6.

3.7 THEOREM. If $G \subset \mathcal{H}(\mathbf{R})$ is abelian and has isolated fixed points then there exists a nontrivial G-invariant Borel measure which is finite on compact sets.

4. Solvable groups and quasi-invariant measures. We now consider the problem of extending an invariant measure from a subgroup of $\mathcal{H}(\mathbf{R})$ to a larger subgroup. If $\mu$ is a measure and $g \in \mathcal{H}(\mathbf{R})$ then the measure $g_{*} \mu$ is defined by $g_{*} \mu(E)=\mu\left(g^{-1}(E)\right)$ for every measurable set $E \subset \mathbf{R}$. The measure $\mu$ is $g$-invariant iff $g_{*} \mu=\mu$. Note that if $g, h \in \mathcal{H}(\mathbf{R})$ then $(g h)_{*} \mu=g_{*}\left(h_{*} \mu\right)$. Suppose $H$ is a normal subgroup of $G \subset$ $\mathcal{H}(\mathbf{R})$. If $\mu$ is an $H$-invariant measure then $g_{*} \mu$ is also $H$-invariant since $\left(g h g^{-1}\right)_{*}\left(g_{*} \mu\right)=(g h)_{*} \mu=g_{*} \mu$ whenever $g \in G, h \in H$. If $H \subset \mathcal{H}^{+}(\mathbf{R})$ and $\tau_{\mu} \in$ $\operatorname{Hom}(H ; \mathbf{R})$ is nonzero then by the proof of 2.1 there is a positive real number $c(g)$ such that $\tau_{g_{*} \mu}=c(g) \tau_{\mu}$. The map $A_{G}: G \rightarrow \mathbf{R}$ defined by $A_{G}(g)=\log c(g)$ is a homomorphism. Note that $A_{G}(g)=0$ iff $\tau_{\mu}(h)= \pm \tau_{\mu}\left(g^{-1} h g\right)$ for all $h \in H$ (since $\tau_{g_{*} \mu}(h)= \pm \tau_{\mu}\left(g^{-1} h g\right)$ with + if $g$ preserves orientation and - if $g$ reverses orientation).

4.1 Lemma. Suppose $H$ is a normal subgroup of $G \subset \mathcal{H}^{+}(\mathbf{R})$ and $\mu$ is a nontrivial $H$-invariant Borel measure which is finite on compact sets. Suppose also that $\tau_{\mu} \neq 0$ in $\operatorname{Hom}(H ; \mathbf{R})$ and $A_{G}=0$ in $\operatorname{Hom}(G ; \mathbf{R})$.

(i) If $\tau_{\mu}(H)$ is dense in $\mathbf{R}$ then $\mu$ is $G$-invariant.

(ii) If $\tau_{\mu}(H)$ is infinite cyclic and $G / H$ is amenable then there exists a G-invariant Borel measure which is finite on compact sets.

Proof. (i) follows immediately from 2.2. Suppose $\tau_{\mu}(H)$ is infinite cyclic. By multiplying $\mu$ by a constant we may suppose $\tau_{\mu}(H)=\mathbf{Z}$. Let $H_{0}=\operatorname{ker} \tau_{\mu}$. Fix $H_{0}$ is a 
nonempty set (since it contains the support of $\mu$ ) which is $H$-invariant and, therefore, unbounded in either direction. Since $H_{0}=\{h \in H \mid \operatorname{Fix}(h) \neq \varnothing\}$ and $\operatorname{Fix}\left(g h g^{-1}\right)=g(\operatorname{Fix}(h)), H_{0}$ is normal in $G$. Thus, we think of $G / H_{0}$ as a group of orientation preserving homeomorphisms of Fix $H_{0}$. We claim that $H / H_{0}$ is in the center of $G / H_{0}$. Let $h_{1} \in H$ be such that $\tau_{\mu}\left(h_{1}\right)=1$. We must show that for any $g \in G$ and $x \in$ Fix $H_{0}, g^{-1} h_{1} g(x)=h_{1}(x)$. If $\nu$ is the $H$-invariant measure with unit mass at each of the points $h_{1}^{n}(x)$ then

$$
\nu\left[x, g^{-1} h_{1} g(x)\right)=\tau_{\nu}\left(g^{-1} h_{1} g\right)=\tau_{g_{*} \nu}\left(h_{1}\right)=\tau_{\nu}\left(h_{1}\right)=\nu\left[x, h_{1}(x)\right)=1
$$

which implies that $g^{-1} h_{1} g(x) \leqslant h_{1}(x)$. On the other hand, if $\nu$ is the $H$-invariant measure with unit mass at each of the points $g^{-1} h_{1}^{n} g(x)$ then a similar argument interchanging $h_{1}$ and $g^{-1} h_{1} g$ yields $h_{1}(x) \leqslant g^{-1} h_{1} g(x)$ so $H / H_{0}$ is in the center of $G / H_{0}$. Now the quotient group $\left(G / H_{0}\right) /\left(H / H_{0}\right) \cong G / H$ acts on the compact quotient space Fix $H_{0} / x \sim h_{1}(x)$ and by 1.1 there is a nontrivial finite invariant Borel measure which determines a $G / H_{0}$-invariant measure on Fix $H_{0}$, i.e., a $G$-invariant measure on $\mathbf{R}$ which is finite on compact sets. This proves (ii) and the proof of 4.1 is complete.

A measure $\mu$ will be called quasi-invariant for $G \subset \mathcal{H}(\mathbf{R})$ if for each $g \in G$ there is a positive constant $c(g)$ such that $g_{*} \mu=c(g) \mu$. (Note that this is more restrictive than requiring that $G$ permutes sets of measure zero.) If $G$ is a subgroup of $\mathcal{H}(\mathbf{R})$ let $G^{+}=G \cap \mathcal{H}^{+}(\mathbf{R}) . G^{+}$is a normal subgroup of index at most 2 in $G$. If $H$ is a normal subgroup of $G \subset \mathcal{H}(\mathbf{R})$ then so is $H^{+}$.

4.2 Lemma. Let $H$ be a normal subgroup of $G \subset \mathcal{H}(\mathbf{R})$ and let $\mu$ be an $H$-invariant Borel measure which is finite on compact sets and such that $\tau_{\mu} \neq 0$ in $\operatorname{Hom}(H ; \mathbf{R})$. If $A_{G} \neq 0$ then $\mu$ is $G$-quasi-invariant.

Proof. For $h \in H^{+}, g \in G$ we have

$$
\tau_{\mu}\left(g^{-1} h g\right)= \pm \tau_{g_{*} \mu}(h)= \pm c(g) \tau(h) \quad(c(g)>0) .
$$

$A_{G} \neq 0$ means that $c(g) \neq 1$ for some $g$ so $\tau_{\mu}\left(H^{+}\right)$is dense in R. From 2.2 it follows that $g_{*} \mu=c(g) \mu$ where $c(g)$ is the constant from the definition of $A_{G}$.

4.3 Lemma. Suppose $H$ is a normal subgroup of $G \subset \mathcal{H}(\mathbf{R})$ and $\mu$ is a nontrivial Borel measure on $\mathbf{R}$ which is finite on compact sets. Suppose $\mu$ is $H$-quasi-invariant and let $H_{0}=\left\{h \in H^{+} \mid h_{*} \mu=\mu\right\}$. If $\tau_{\mu}: H_{0} \rightarrow \mathbf{R}$ is nonzero and $A_{H} \neq 0$ then $\mu$ is G-quasi-invariant.

Proof. Since $\tau_{\mu}$ is a nonzero element of $\operatorname{Hom}\left(H_{0} ; \mathbf{R}\right)$ it follows that $h \in H_{0}$ iff either $\operatorname{Fix}(h)=\varnothing\left(\tau_{\mu}(h) \neq 0\right)$ or sup $\operatorname{Fix}(h)=+\infty$ and inf $\operatorname{Fix}(h)=-\infty\left(\tau_{\mu}(h)=\right.$ $0)$. Since $\operatorname{Fix}\left(g h g^{-1}\right)=g(\operatorname{Fix}(h))$ and $H$ is normal in $G$ we conclude that $H_{0}$ is normal in $G$. Since $A_{H} \neq 0$ we have $A_{G} \neq 0$ and 4.3 follows from 4.2.

We are now ready to consider the problem of existence of quasi-invariant measures for solvable subgroups of $\mathcal{H}(\mathbf{R})$. A group $G$ is solvable if there is a series

$$
1=G_{0} \subset G_{1} \subset \cdots \subset G_{n}=G
$$


such that $G_{i-1}$ is a normal subgroup of $G_{i}, i=1, \ldots, n$, and $G_{i} / G_{i-1}$ is abelian. If each $G_{i} / G_{i-1}$ is (finite or infinite) cyclic, $G$ is called polycyclic. A solvable group is polycyclic iff every subgroup is finitely generated [14]. Denote by $\mathcal{S}$ the class of groups such that $G \in \mathcal{S}$ iff there is a normal series as above except that each quotient $G_{i} / G_{i-1}$ satisfies the following condition: There is a finitely generated subgroup $H_{i} \subset G_{i} / G_{i-1}$ which has nonexponential growth $[9,14]$ and whenever $g \in G_{i} / G_{i-1}$ there is a natural number $m$ such that $g^{m} \in H_{i}$. Clearly, $\delta$ includes polycyclic groups and finitely generated groups having nonexponential growth. Also included in $\delta$ are all groups with the property that each $G_{i} / G_{i-1}$ is either finitely generated with nonexponential growth or isomorphic to a subgroup of $\mathbf{Q}$. On the other hand, $\delta$ does not include all finitely generated groups (e.g., the group $G$ of $\S 5$ ).

4.4 Theorem. Suppose $G \subset \mathcal{H}(\mathbf{R})$ is solvable with normal series $1=G_{0} \subset G_{1} \subset$ $\cdots \subset G_{n}=G$ such that each $G_{i}$ is boundedly generated. Then there exists a nontrivial Borel measure on $\mathbf{R}$ which is $G$-quasi-invariant and finite on compact sets.

4.5 Corollary. If $G \subset \mathcal{H}(\mathbf{R})$ is in $\varsigma$ then there exists a nontrivial $G$-quasi-invariant measure which is finite on compact sets.

Proof OF 4.4. If $x \in$ Fix $G^{+}$then the measure $\mu^{+}$with unit mass at $x$ is finite and if $g \in G-G^{+}$then $\mu^{+}+g_{*} \mu^{+}$is $G$-invariant so we assume Fix $G^{+}=\varnothing$. Let $j<n$ be the largest integer such that Fix $G_{j}^{+} \neq \varnothing$. Since $G_{j}^{+}$is normal in $G_{j+1}$, Fix $G_{j}^{+}$is $G_{j+1}$-invariant and there is induced an action of $G_{j+1} / G_{j}^{+}$on Fix $G_{j}^{+}$. According to 3.3 and Remark (1) after 3.6 there is a $G_{j+1}$-invariant measure $\mu$ on $\mathbf{R}$ which is finite on compact sets. Since Fix $G_{j+1}^{+}=\varnothing, \tau_{\mu} \in \operatorname{Hom}\left(G_{j+1}^{+} ; \mathbf{R}\right)$ is nonzero by 1.2. If $G$ has a nontrivial invariant measure we are done, so suppose otherwise. Let $k<n$ be the largest integer such that $G_{k}$ has a nontrivial invariant Borel measure which is finite on compact sets. Denote such a measure by $\mu$. Since Fix $G_{k}=\varnothing, \tau_{\mu} \neq 0$ in $\operatorname{Hom}\left(G_{k}^{+} ; \mathbf{R}\right)$. According to 4.1 we must have $A_{G_{k+1}} \neq 0$ (since $A_{G_{k+1}}^{+} \neq 0$ ) and by 4.2, $\mu$ is $G_{k+1}$-quasi-invariant. Now suppose that $l>k$ is such that $l<n$ and $\mu$ is $G_{r}$-quasi-invariant. By $4.3, \mu$ is $G_{l+1}$-quasi-invariant. Therefore, $\mu$ is $G$-quasiinvariant and the proof of 4.4 is complete.

Proof OF 4.5. The only place in the proof of 4.4 where we needed to know that a subgroup of $G$ was boundedly generated was in going from $G_{j}$ (Fix $G_{j}^{+} \neq \varnothing$ ) to $G_{j+1}$ $\left(\right.$ Fix $\left.G_{j+1}^{+}=\varnothing\right)$. Since every element of $G_{j+1}^{+} / G_{j}^{+}$has some positive power in a finitely generated subgroup and acts on Fix $G_{j}^{+}, G_{j+1}^{+}$(and $G_{j+1}$, therefore) is boundedly generated.

REMARKs. (1) If a quasi-invariant $\mu$ is $G$-invariant and Fix $G=\varnothing$ then $\tau_{\mu} \neq 0$ in $\operatorname{Hom}\left(G^{+} ; \mathbf{R}\right)$ and if $\mu$ is not $G$-invariant then $A_{G} \neq 0$ in $\operatorname{Hom}(G ; \mathbf{R})$ (even if Fix $G \neq \varnothing)$.

(2) 4.4 applies to some solvable groups which are not in $\mathcal{S}$. For example, if $G \subset \operatorname{Diff}^{2}(\mathbf{R})$ has (arbitrary) abelian commutator subgroup $H$ and $G / H$ is finitely generated then $G$ has a nontrivial quasi-invariant measure by 3.6 and the remark following its proof. 
4.6 CoRollary. Suppose $G \subset \mathcal{H}(\mathbf{R})$ satisfies the hypotheses of 4.4 or of 4.5 . If $G$ is minimal (i.e., $G(x)$ is dense in $\mathbf{R}$ for every $x \in \mathbf{R}$ ) then $G$ is conjugate (in $\mathcal{H}(\mathbf{R})$ ) to a subgroup of the affine group.

Proof. If $\mu$ is a $G$-quasi-invariant measure then its support is $G$-invariant and, hence, all of $\mathbf{R}$. Since $\mu$ has no atoms the map $h: \mathbf{R} \rightarrow \mathbf{R}$ defined by

$$
h(x)=\left\{\begin{array}{cc}
\mu([0, x]), & x \geqslant 0, \\
-\mu([x, 0]), & x \leqslant 0,
\end{array}\right.
$$

is a homeomorphism and $h G h^{-1}$ is a subgroup of the affine group.

REMARK. The conclusion of 4.6 holds for any minimal $G$ which has a quasiinvariant measure. When the measure has no atoms but $G$ is not minimal then, as in the proof of 2.2, we obtain a semiconjugacy for some quotient group.

4.7 THEOREM. If $G \subset \mathcal{H}(\mathbf{R})$ is a solvable group with isolated fixed points then there exists a G-quasi-invariant Borel measure which is finite on compact sets.

Proof. Suppose $G$ has normal series

$$
1=G_{0} \subset G_{1} \subset \cdots \subset G_{n}=G .
$$

By 3.7 there is a $G_{1}$-invariant measure. If $G$ has an invariant measure we are done, so assume otherwise and let $k<n$ be the largest integer such that $G_{k}$ has a nontrivial invariant Borel measure which is finite on compact sets. Denote such a measure by $\mu$. Consider the discrete set Fix $G_{k}^{+}$. If Fix $G_{k}^{+} \neq \varnothing$ then setting $I_{x}=\left\{g \in G_{k+1} \mid\right.$ $g(x)=x\}$ we would have $G_{k+1} / I_{x}$ either trivial or infinite cyclic for $x \in$ Fix $G_{k}^{+}$, which would imply the existence of a $G_{k+1}$-invariant measure (with atoms!). So Fix $G_{k}^{+}=\varnothing$ and $\tau_{\mu} \neq 0$ in $H\left(G_{k}^{+}, \mathbf{R}\right)$. Let $H=\operatorname{ker} \tau_{\mu} . H$ is normal in $G_{k+1}$ and Fix $H \neq \varnothing$. According to 4.1 we conclude that $A_{G_{k+1}} \neq 0$ and, therefore, by $4.2, \mu$ is $G_{k+1}$-quasi-invariant. By $4.3, \mu$ is $G$-quasi-invariant and the proof of 4.7 is complete.

4.8 Proposition. Suppose $G \subset \mathcal{H}(\mathbf{R})$ is a group and $H \subset G$ a nontrivial normal fixed point free subgroup which is not infinite cyclic. Then $G$ has a quasi-invariant Borel measure which is finite on compact sets.

Proof. By 3.2 there is a nontrivial $H$-invariant Borel measure $\mu$ which is finite on compact sets. Since $H$ is fixed point free, $\tau_{\mu}: H \rightarrow \mathbf{R}$ is injective and, therefore $\tau_{\mu}(H)$ is dense in $\mathbf{R}$. By 4.1 and $4.2, \mu$ is $G$-quasi-invariant.

5. An example. In this section, we describe an example to illustrate the fact that various hypotheses made earlier are not frivolous.

Let $f: \mathbf{R} \rightarrow \mathbf{R}$ be an orientation preserving expanding homeomorphism with unique fixed point 0 . That is, $f(0)=0$ and if $I_{0}=\left[a_{0}, b_{0}\right]$ is an interval with 0 in its interior, we have $f\left(I_{0}\right) \supset I_{0}, \cup f^{n}\left(I_{0}\right)=\mathbf{R}, \cap f^{n}\left(I_{0}\right)=\{0\}$. Denote $f^{n}\left(I_{0}\right)$ by $I_{n}$, $n \in \mathbf{Z}$, and denote by $a_{n}, b_{n}$ the endpoints of $I_{n}\left(a_{n}<b_{n}, a_{n}=f^{n}\left(a_{0}\right), b_{n}=f^{n}\left(b_{0}\right)\right)$. Let $g: I_{0} \rightarrow I_{0}$ be an orientation preserving homeomorphism such that $g(x)>x$ when $a_{0}<x<b_{0}$ and $g\left(a_{-1}\right)=b_{-1}$. Define $g_{n}: I_{n} \rightarrow I_{n}$ by $g_{n}=f^{n} g f^{-n}$. We now extend the definition of $g_{n}$ to all of $\mathbf{R}$. 
Suppose $g_{i}, 0 \leqslant i \leqslant l-1$, can be extended to $I_{l-1}$ in such a way that $g_{i}$ and $g_{j}$ commute and $g_{j}=f^{j-i} g_{i} f^{i-j}$ whenever $0 \leqslant i \leqslant j \leqslant l-1$. We have

$$
\text { int } I_{l}=\bigcup_{m} g_{l}^{m}\left(I_{l-1}\right)
$$

where consecutive intervals $g_{l}^{m}\left(I_{l-1}\right)$ and $g_{l}^{m+1}\left(I_{l-1}\right)$ intersect only at a common endpoint. Define

$$
g_{i}(x)=g_{l}^{m} g_{i} g_{l}^{-m}(x) \text { when } x \in g_{l}^{m}\left(I_{l-1}\right) .
$$

This gives a well-defined extension of $g_{i}(0 \leqslant i \leqslant l-1)$ to $I_{l}$ such that $g_{i}$ and $g_{j}$ commute and $g_{j}=f^{j-1} g_{i} f^{i-j}$ whenever $0 \leqslant i \leqslant j \leqslant l-1$. In order to show that these relations hold when $0 \leqslant i \leqslant j \leqslant l$ it suffices to show that

$$
f^{i-l} g_{l} f^{l-i} g_{l}^{m}=g_{l}^{m} g_{i} \text { for all } m \text {. }
$$

We obtain $g_{l}^{m} g_{i}=f^{i} g_{l-i}^{m} g f^{-i}$ and $f^{i-l} g_{l} f^{l-i} g_{l}^{m}=f^{i} g g_{l-i}^{m} f^{-i}$ but these agree since $g$ $\left(=g_{0}\right)$ and $g_{l-i}$ commute on $I_{0}$. Extending inductively, we obtain elements $g_{n} \in$ $\mathcal{H}^{+}(\mathbf{R})$ for each $n \in \mathbf{Z}$ such that $g_{i}$ and $g_{j}$ commute for all $i, j$ and $g_{i+1}=f g_{i} f^{-1}$. The group $H$ generated by $\left\{g_{i}\right\}$ is an abelian normal subgroup of the solvable group $G$ generated by $f$ and $g\left(=g_{0}\right)$. We claim that $H$ (and, therefore, $G$ ) has no quasiinvariant measure which is finite on compact sets.

Suppose $\mu$ is a $H$-quasi-invariant measure. If $\mu$ is nontrivial then for some $n, \mu\left(\left[a_{n}, b_{n}\right)\right)>0$. If $\mu$ is $H$-quasi-invariant, then there is a constant $c>0$ such that $\mu\left(g_{n+1}\left(\left[a_{n}, b_{n}\right)\right)\right)=c \mu\left(\left[a_{n}, b_{n}\right)\right)$. Therefore, by countable additivity, since $\left[a_{n+1}, b_{n+1}\right)=\cup_{m} g_{n+1}^{m}\left(\left[a_{n}, b_{n}\right)\right)$ (disjoint union), we obtain

$$
\mu\left(\left[a_{n+1}, b_{n+1}\right)\right)=\mu\left(\left[a_{n}, b_{n}\right)\right) \sum_{-\infty}^{\infty} c^{m}=\infty
$$

so $\mu\left(I_{n+1}\right)=\infty$ and $\mu$ is not finite on compact sets.

6. Codimension one foliations covered by an R-foliation. In this section we consider a manifold $M$ with a continuous codimension one foliation $\mathscr{F}$. The leaf space of $\mathscr{F}$ is, in general, not Hausdorff. If the leaf space of $\mathscr{F}$ is homeomorphic to $\mathbf{R}$ we say that $\mathscr{F}$ is an R-foliation. If $\tilde{M}$ is the universal covering space of $M$ we denote by $\tilde{\mathscr{F}}$ the codimension one foliation of $\tilde{M}$ induced by the covering projection from $\tilde{\mathscr{F}}$. If the leaf space of $\tilde{\mathscr{F}}$ is homeomorphic to $\mathbf{R}$ we say $\mathscr{F}$ is covered by an $\mathbf{R}$-foliation. The fundamental group of $M$ acts on $\tilde{M}$ and preserves $\tilde{\mathscr{F}}$. In this manner we obtain a homomorphism $h: \pi_{1}(M) \rightarrow \mathcal{H}(\mathbf{R})$ whose image is called the holonomy group of $\mathscr{F}$ and will be denoted by $H(\mathscr{F})$. It is defined only up to conjugacy in $\mathcal{H}(\mathbf{R})$ since it depends on the homeomorphism between $\mathbf{R}$ and the leaf space of $\tilde{\mathscr{F}}$. (Because : of conflicting conventions $h$ usually turns out to be an antihomomorphism but $H(\mathscr{F})=$ $h\left(\pi_{1}(M)\right)$ is still a group.) The holonomy group of $\mathscr{F}$ contains much information about $\mathscr{F}$. For example, if $H(\mathscr{F})$ is (conjugate to) a subgroup of the affine group then $\mathcal{F}$ has a transverse affine structure in the sense of [4]. Of course, if the conjugacy to a subgroup of the affine group is not differentiable then the affine structure is not differentiably equivalent to the original differential structure. $\mathscr{F}$ is minimal, i.e., every leaf of $\mathscr{F}$ is dense in $M$, iff $H(\mathscr{F})$ acts minimally on $\mathbf{R} . H(\mathscr{F})$ is a fixed point free group iff every leaf of $\mathscr{F}$ has trivial holonomy group. 
6.1 THEOREM. Let $\mathscr{F}$ be a codimension one foliation of a manifold $M$ which is covered by an R-foliation $\tilde{\mathcal{F}}$ of $\tilde{M}$ and suppose at least one of the following is satisfied.

(i) Every leaf of $\mathscr{F}$ has trivial holonomy group.

(ii) $\mathcal{F}$ is of class $C^{2}$ and $H(\mathcal{F})$ is abelian.

(iii) $H(\mathscr{F})$ is abelian and has isolated fixed points.

Then there is a nontrivial $H(\mathscr{F})$-invariant measure which is finite on compact sets. Furthermore, if $\mathscr{F}$ is transversely oriented (automatic for (i)) and $H(\mathscr{F})$ is nontrivial then $H^{1}(M ; \mathbf{R}) \neq 0$.

Proof. The first conclusion in cases (i), (ii), (iii) follows from 3.2, 3.6, 3.7, respectively. When $\mathscr{F}$ is transversely oriented $H(\mathscr{F}) \subset \mathcal{H}^{+}(\mathbf{R})$. If Fix $H(\mathscr{F})=\varnothing$ then $\tau_{\mu} \neq 0$ in $\operatorname{Hom}(H(\mathscr{F}) ; \mathbf{R})$ so $\tau_{\mu} h \in \operatorname{Hom}\left(\pi_{1}(M) ; \mathbf{R}\right)$ represents a nonzero element of $H^{1}(M ; \mathbf{R})$. If Fix $H(\mathscr{F}) \neq \varnothing$ we restrict the action of $H(\mathscr{F})$ to a maximal open interval of $\mathbf{R}-$ Fix $H(\mathscr{F})$. Since an open interval is homeomorphic to $\mathbf{R}$ we obtain as before (using (i), (ii), or (iii) to obtain a new $\mu$ ) a nonzero element of $\operatorname{Hom}\left(\pi_{1}(M) ; \mathbf{R}\right)$ and 6.1 is proved.

REMARKs. (1) If, in addition, $\mathscr{F}$ (transversely oriented) is minimal in 6.1 then $H(\mathscr{F})$ is conjugate to a group of translations of $\mathbf{R}$.

(2) Examples in [10] show that transverse orientability is essential for the last conclusion of 6.1 .

(3) When $M$ is compact and $\mathscr{F}$ is $C^{2}$ and satisfies (i) it is well known that $\mathscr{F}$ is covered by an $\mathbf{R}$-foliation (actually $\tilde{M} \approx \tilde{L} \times \mathbf{R}$ where $\tilde{L}$ is the universal covering of any leaf $L$ of $\mathscr{F}$ ). For $M$ compact but $\mathscr{F}$ not $C^{2}$ it seems to be an open question whether $\mathscr{F}$ must be covered by an $\mathbf{R}$-foliation if (i) is satisfied.

6.2 THEOREM. Let $\mathcal{F}$ be a codimension one foliation of a manifold $M$ which is covered by an R-foliation $\tilde{\mathscr{F}}$ of $\tilde{M}$ and suppose at least one of the following is satisfied.

(i) $H(\mathscr{F})$ is in $\mathcal{S}$.

(ii) $H(\mathscr{F})$ is finitely generated with abelian commutator subgroup and $\mathscr{F}$ is $C^{2}$.

(iii) $\pi_{1}(M)$ is solvable, $M$ is compact, and $\tilde{M}$ is contractible.

(iv) $H(\mathscr{F})$ is solvable and has isolated fixed points (e.g., if $\mathscr{F}$ is real analytic).

(v) $H(\mathscr{F})$ has a nontrivial normal fixed point free subgroup which is not infinite cyclic.

Then there is a nontrivial $H(\mathcal{F})$-quasi-invariant measure $\mu$ which is finite on compact sets. Furthermore:

(a) If $\mathscr{F}$ is also minimal then $\mathscr{F}$ has a transverse affine structure.

(b) If $\mu$ is not $H(\mathscr{F})$-invariant then $H^{1}(M ; \mathbf{R}) \neq 0$.

(c) If $\mu$ is $H(\mathscr{F})$-invariant, $H(\mathscr{F})$ is nontrivial, and $\mathscr{F}$ is transversely oriented then $H^{\mathrm{l}}(M ; \mathbf{R}) \neq 0$.

Proof. The first conclusion in cases (i), (iv), (v) follows from 4.5, 4.7 4.8, respectively. In case (ii) the conclusion follows from Remark (2) after 4.5. In case (iii), $\pi_{1}(M)$ is polycyclic by (3.3) of [1] so $H(\mathscr{F})$ is polycyclic and the conclusion follows from case (i). Now (a) follows from the remark after 4.6, (b) follows from Remark (1) after 4.5, and (c) follows as in the proof of 6.1. This proves 6.2. 
REMARK. It is necessary to assume that the subgroup in case (v) is not infinite cyclic. For example, consider the stable manifold foliation of the geodesic (Anosov) flow on the unit tangent bundle of a compact surface $S$ with negative curvature. The circle fibers represent elements in the center of $\pi_{1}\left(T_{1} S\right)$ and the center acts without fixed points on $\mathbf{R} \approx$ leaf space in universal covering. Even though the foliation of $T_{1} S$ is minimal it can be shown that it has no transverse affine structure. In fact, it can be shown that the only stable manifold foliations of Anosov flows which have smooth transverse affine structures arise from suspensions of Anosov diffeomorphisms and geodesic flows cannot be suspensions $[8,11]$. For codimension one Anosov flows this is true even for continuous affine structures. This latter observation is proved in [11] (using results of this paper) with an additional hypothesis. However, using a more recent suspension criterion due to Fried (Theorem I of [3]) the general case is immediate. In particular, the conjecture of Verjovsky is proved.

TheOREM. If $\phi_{t}: M \rightarrow M$ is a codimension one Anosov flow and $\pi_{1}(M)$ is solvable then $\phi_{t}$ is conjugate (not necessarily preserving parameters) to the suspension of a hyperbolic toral automorphism.

7. Reeb stability for continuous codimension one foliations. Suppose $L$ is a compact leaf of a transversely oriented codimension one foliation $\mathscr{F}$. To what extent does the topology of $L$ determine the structure of $\mathcal{F}$ in a neighborhood of $L$ ? For foliations of class $C^{1}$ Thurston's generalization of the Reeb Stability Theorem [13] says that if $H^{1}(L ; \mathbf{R})=0$ then there is a neighborhood of $L$ such that the restriction of $\mathcal{F}$ to the neighborhood is homeomorphic to the foliation of $L \times \mathbf{R}$ having leaves $L \times\{t\}$. It is also observed in [13] that this result does not extend to $C^{0}$ foliations. In [9] it is shown that the same conclusion holds for $C^{0}$ foliations if we make the additional assumption that $\pi_{1}(M)$ has nonexponential growth. (Note: (8.3) of [9] is not stated correctly. $H(L)$ and $\operatorname{Hom}(H(L) ; \mathbf{R})$ should be changed to $\pi_{1}(L)$ and $H^{1}(L ; \mathbf{R})$, respectively.) The methods of [9] involved finding an invariant measure for a certain pseudogroup acting on an interval. In general, the considerations of $\$ 4$ of the present paper do not extend to pseudogroups. The reason is that the definition of a pseudogroup allows for compositions by extension, i.e., if $\gamma_{1}, \gamma_{2}$ are defined on open sets $U_{1}$ and $U_{2}$, respectively, and agree on $U_{1} \cap U_{2}$ then we define $\gamma_{1} \cup \gamma_{2}$ to be $\gamma_{1}$ on $U_{1}$ and $\gamma_{2}$ on $U_{2}$. If $\mu$ is a measure such that $\gamma_{1 *} \mu=c_{1} \mu, \gamma_{2 *} \mu=c_{2} \mu$ with $c_{2} \neq c_{2}$ (this could easily happen if $\operatorname{supp} \mu \cap U_{1} \cap U_{2}=\varnothing$ ) then $\mu$ would not be quasi-invariant for $\gamma_{1} \cup \gamma_{2}$. However, in our situation we may restrict the definition of a pseudogroup as follows: Assume that our pseudogroup acts on an interval $(0, \varepsilon)$ and every element has domain of the form $(0, \alpha)$ where $\alpha \leqslant \varepsilon$ depends on the element chosen. In other words, we only allow restrictions to intervals which have 0 as left endpoint. As in [9] we define the translation number homomorphism of an invariant measure for such a pseudogroup. The arguments used earlier in this paper may be modified in the obvious manner to obtain the following Reeb stability results which parallel (8.2) and (8.3) of [9].

7.1 ThEOREM. Let $L$ be a compact leaf in a continuous transversely oriented codimension one foliation $\mathcal{F}$ and denote by $H(L)$ the holonomy group of $L$. Suppose that 
$H(L)$ is in $\delta$. Then $\operatorname{Hom}(H(L) ; \mathbf{R})=0$ if, and only if, $H(L)=0$. In particular, if $\pi_{1}(L)$ is in $\delta$ and $H^{1}(L ; \mathbf{R})=0$ then $H(L)=0$ and there is a neighborhood of $L$ such that the restriction of $\mathscr{F}$ to the neighborhood is homeomorphic to the foliation of $L \times \mathbf{R}$ having leaves $L \times\{t\}$.

7.2 Corollary. Suppose $\mathscr{F}$ is a continuous transversely oriented codimension one foliation of a compact manifold $M$. If $L$ is a compact leaf of $\mathscr{F}$ such that $\pi_{1}(L)$ is in $\mathcal{S}$ and $H^{1}(L ; \mathbf{R})=0$ then every leaf of $\mathscr{F}$ is homeomorphic to $L$ and, furthermore, $M$ is a bundle over the circle whose fibers are the leaves of $\mathcal{F}$.

REMARKs. (1) If $\mathscr{F}$ is determined by a locally free action of a solvable Lie group then every leaf of $\mathscr{F}$ has polycyclic fundamental group and, therefore, polycyclic holonomy group.

(2) If $\pi_{1}(L)$ is solvable and $L$ has contractible universal covering space then $\pi_{1}(L)$ (and, therefore, $H(L)$ ) is polycyclic [1].

(3) The proofs of 7.1 and 7.2 use quasi-invariant measures to construct elements of $\operatorname{Hom}(H(L) ; \mathbf{R})$. Since there may be other ways to get such homomorphisms, the question of whether 7.1 and 7.2 can be extended to arbitrary solvable groups remains open.

(4) Some applications of the techniques in this paper to global stability questions are given in [12].

\section{REFERENCES}

1. R. Bieri, Gruppen mit Poincaré-Dualität, Comment. Math. Helv. 47 (1972), 373-396.

2. G. Birkhoff, Lattice theory, Amer. Math. Soc. Colloq. Publ., vol. 25, Amer. Math. Soc., Providence, R.I., 1948.

3. D. Fried, The geometry of cross-sections to flows, Topology 21 (1982), 353-371.

4. P. Furness and E. Fédida, Transversally affine foliations, Glasgow Math. J. 17 (1976), 106-111.

5. S. Goodman and J. Plante, Holonomy and averaging in foliated sets, J. Differential Geometry 14 (1979), 401-407.

6. F. Greenleaf, Invariant means on topological groups, Van Nostrand, New York, 1969.

7. N. Kopell, Commuting diffeomorphisms, Proc. Sympos. Pure Math., vol. 14, Amer. Math. Soc., Providence, R.I., 1969, pp. 165-184.

8. J. Plante, Anosov flows, Amer. J. Math. 94 (1972), 729-754.

9. , Foliations with measure preserving holonomy, Ann. of Math. (2) 102 (1975), 327-361.

10. Foliations of 3-manifolds with solvable fundamental group, Invent. Math. 51 (1979), 219-230

11. Anosov flows, transversely affine foliations, and a conjecture of Verjovsky, J. London Math. Soc. 23 (1981), 359-362.

12. Stability of codimension one foliations by compact leaves, Topology (to appear).

13. W. Thurston, A generalization of the Reeb Stability Theorem, Topology 13 (1974), 347-352.

14. J. Wolf, Growth of finitely generated solvable groups and curvature of Riemannian manifolds, J. Differential Geometry 2 (1968), 421-446.

Department of Mathematics, University of North Carolina, Chapel Hill, North Carolina 27514 